EPJ Web of Conferences 61, 04006 (2013)

DOI: 10.1051/epjconf/ 20136104006

(C) Owned by the authors, published by EDP Sciences, 2013

\title{
Jet evolution in Steep Spectrum Radio Quasars: a multiwavelength study.
}

\author{
Eleonora Torresi ${ }^{1}$,a , Paola Grandi ${ }^{1}$, and the TANGO team on behalf of the Fermi-LAT Collaboration \\ ${ }^{1}$ Istituto Nazionale di Astrofisica-IASFBO, Via Gobetti 101, I-40129, Bologna, Italy
}

\begin{abstract}
Thanks to the Fermi $\gamma$-ray satellite, it is now confirmed that Misaligned Active Galactic Nuclei (MAGN), i.e. radio galaxies and steep spectrum radio quasars, are a new class of $\mathrm{GeV}$ emitters. In this work we present the first $\gamma$-ray and multiwavelength study of the two steep spectrum radio quasars, i.e. 3C 207 and 3C 380, belonging to the MAGN sample. From the $\gamma$-ray variability study we estimate the physical size of the zone where high-energy photons are dissipated: for both sources this region should be very compact, not larger than 0.05 pc. As a successive step, we build multiwavelength light curves of 3C 207 and 3C 380 to search for possible simultaneous outbursts in different wavebands with the aim of localizing the compact emitting region. This is an important issue with strong impact on theoretical models: indeed, knowing where highenergy photons are dissipated (at sub-pc or pc-scale) provides information on the nature of the seed photons involved in the production of the $\mathrm{GeV}$ radiation.
\end{abstract}

\section{Introduction}

The Fermi $\gamma$-ray satellite has given an invaluable contribution to the discovery of Misaligned AGN (MAGN) as a new class of GeV sources. MAGN are Radio-Loud objects [FRI and FRII radio galaxies [1] and Steep Spectrum Radio Quasars (SSRQ)] that are characterized by steep radio spectra $\left(\alpha_{\mathrm{r}}>0.5\right)$ and resolved and possibly symmetrical radio structures. In these sources the jet is pointing at large inclination angles from the observer's line of sight $\left(\theta \gtrsim 10^{\circ}\right)$, implying a small Doppler factor $(\delta)$ and, consequently, the jet radiation is not strongly boosted (see also [2], [3]). For this reason MAGN were not considered ideal GeV targets, but the Fermi Large Area Telescope (LAT; [4]) detection of 11 sources (6 FRI, 2 FRII, 2 SSRQ and 3C $120^{1}$ ) in 15 months of survey [5] has changed this view. Despite their small number, MAGN are extremely appealing as they offer the possibility to study high-energy non-thermal phenomena from a different perspective with respect to blazars.

One challenging aspect of the high-energy study of MAGN, and of blazars in general, is the localization of the $\gamma$-ray emitting region. Numerous studies performed on blazars have pointed out that the location of the $\gamma$-ray emitting region is not unique: $\gamma$-ray photons can be dissipated on sub-pc scale, within the broad line region (BLR) as reported, e.g. by [6], [7] or outside of it, on pc scales ([8], [9], [10], [11], [12], [13], [14]). Different locations imply different seed photons involved in the Inverse Compton process (leptonic models) that produces photons at energies above $100 \mathrm{MeV}$. Optical/UV photons are possibly the dominant population on sub-pc scale, while outside of the

\footnotetext{
ae-mail: torresi@iasfbo.inaf.it

${ }^{1} 3 \mathrm{C} 120$ is a peculiar FRI characterized by a powerful accretion disk.
}

BLR infrared photons from the torus are probably more influential. In MAGN extended radio lobes at kpc scale can be the origin of $\gamma$-ray emission as well, as in the case of the FRI radio galaxy Centaurus A [15].

$\gamma$-ray variability studies alone can provide information on the size of the emitting region but not on its localization. To this aim multiwavelength campaigns are necessary. This was further demonstrated by the first radio-to$\gamma$-ray study of the FRII radio source 3C 111 [16]. The comparison between the 24-month $\gamma$-ray light curve and mm-optical-X-ray data [17] revealed a simultaneous multiwavelength outburst in late 2008. The short time interval of the GeV activity (approximately two months) indicated that the region is compact with a radius not larger than 0.1 pc, excluding the extended lobes as possible sources of $\gamma$ ray photons. Finally, a deep investigation of the VLBA $43 \mathrm{GHz}$ images of 3C 111 at different epochs, before and after the flare, has allowed us to identify the event producing the outburst, i.e. the ejection of a new radio component in late 2008. Therefore the $\gamma$-ray source was localized in the radio core region at a distance of about $\sim 0.3 \mathrm{pc}$ from the black hole (for a comprehensive discussion see [16]).

After this successful result, we decided to organize a multifrequency monitoring campaign of a sample of MAGN, in order to follow the temporal variability of their jets from radio-to- $\gamma$-rays. This project, called TANGO (Timing Analysis of Non blazar Gamma-ray Objects) ${ }^{2}$, started on February 2012 and is still ongoing. Our monitored sample consists of 20 targets selected from the 3CRR [18] and 2Jy [19] catalogs. Some sources already have a $\mathrm{GeV} \gamma$-ray counterpart, while others are potential $\gamma$-ray candidates. This campaign involves both ground-based

\footnotetext{
${ }^{2}$ For more information please visit the following URL https://hangar.iasfbo.inaf.it/tango/index.html
} 
and space telescopes and covers most of the electromagnetic spectrum from radio frequencies to higher energies. As an example of the potential of this observational approach, we present the preliminary $\gamma$-ray and multiwavelength study of 3C 207 and 3C 380, the only two SSRQ of the MAGN sample.

$3 \mathrm{C} 207$ is a powerful radio source at redshift $\mathrm{z}=0.680$ [20]. VLA images at $8.4 \mathrm{GHz}$ show a double-lobed structure with a prominent jet pointing at the eastern lobe and terminating in a hot-spot ([21]). The Chandra X-ray images of this source resemble this structure with a central nucleus, a western radio lobe, a knot along the jet and a hot-spot [22].

3C 380 is another powerful FRII/SSRQ at $\mathrm{z}=0.692$, originally listed as a compact steep spectrum source (CSS; [23]). However, its largest linear size of $56.8 \mathrm{kpc}$ [24] makes it difficult to reconcile the characteristics of $3 \mathrm{C} 380$ with the definition of a CSS object ${ }^{3}$. The small jet inclination angle $\left(\theta \approx 7^{\circ}\right)$ measured by [26] suggests that this source could be a borderline FRII seen close to the lineof-sight [27]. On parsec scales the jet of 3C 380 appears bent, while on kpc scales it is characterized by two bright knots also visible in the optical band [28].

For both sources superluminal motion of the jet components has been measured (10.75c for 3C 207 and $13.07 \mathrm{c}$ for 3C 380; see [31] for a review).

\section{Gamma-ray variability analysis}

We produced 0.1-100 GeV light curves of 3C 207 and 3C 380 considering 51 months of survey (2008 August 42012 November 4) and using a bin time of 3 months. The Fermi-LAT ScienceTools software (version v9r27p1) was utilized with the P7V6 set of instrument response functions, and using standard procedures. The data analysis was performed exploiting the unbinned maximum likelihood analysis implemented in the gtlike tool. The model for which the unbinned likelihood was calculated is a combination of point-like and diffuse sources located within a region of interest $(\mathrm{RoI})$ of $15^{\circ}$ radius and centered on the source. The spectral slope of each point-like source within this region was fixed to the best-fit value provided by the 2FGL catalog [29], while the normalization was let free to vary. For the sources falling within $15^{\circ}$ and $20^{\circ}$ from the target, normalizations were also fixed to the 2FGL values. The spectral model used for both sources is a power law $\left(\mathrm{dN} / \mathrm{dE}=\mathrm{kE}^{-\Gamma}\right)$ with the spectral slope fixed to the 2FGL value ( $\Gamma=2.35$ for both 3 C 207 and 3 C 380). The significance of the detection was estimated using the Test Statistic (TS) ${ }^{4}$ : when $\mathrm{TS}<10$ the flux value was replaced by a $2 \sigma$ upper limit.

Figure 1 (left panel) shows the 51-month light curve of 3C 207. The source was detected by Fermi only during the first year of operations. For the rest of the time it

\footnotetext{
${ }^{3}$ By definition a CSS object is a radio source not greater than $20 \mathrm{kpc}$ and not exceeding the host galaxy size [25].

${ }^{4}$ The Test Statistic (TS) is defined as $\mathrm{TS}=2\left(\log \mathrm{L}_{0}-\log \mathrm{L}_{1}\right)$, where $\mathrm{L}_{0}$ and $L_{1}$ are the likelihood values when models with and without the source are considered [30].
}

is below the LAT sensitivity threshold. As next step, we generated a light curve with a denser time sampling for the first nine months of data (from 2008 August 4 to 2009 May 4 ), in order to better constrain the shape of the $\gamma$-ray flare. To this aim we applied a "sliding window" method that approximately searches for the maximum TS value using different window sizes and time steps. We found that 1 week is the best minimum bin time for 3C 207 in order to achieve a detection value which is at least $3 \sigma$ above the upper limit values (Figure 1 right panel). The most prominent flare occurred between 2008 September 20 to October 4 , when the source experienced an abrupt flux increase in only 7 days, becoming detectable by the LAT. The same procedure was followed to analyze the $\gamma$-ray light curve of $3 \mathrm{C} 380$. Contrarily to $3 \mathrm{C} 207$, the source was in a quiescent state during the first year of LAT operations while, after that, it is always above the LAT sensitivity threshold (Figure 2 left panel). A thicker sampling of the light curve (starting from the second flare), produced once again with the "sliding window" method, evidenced that a bin time of 15 days is the minimum interval for which the source is detected with a significance greater than $3 \sigma$ (Figure 2 right panel).

\subsection{Gamma-ray variability results}

The size of the emitting region can be estimated from the source variability in the $\gamma$-ray band. Indeed, knowing the time interval of the $\mathrm{GeV}$ flare $(\Delta t)$, on the basis of causality arguments, the emitting region cannot be larger than

$$
R \leq \frac{\Delta t \delta c}{(1+z)}
$$

where $\delta$ is the Doppler factor and $\mathrm{z}$ the redshift of the source. As for 3C 207, the Doppler factor is unknown, however from the superluminal motion [31] we can roughly estimate $\delta \leq 13$. Therefore, on the basis of eq. (1) and considering a time interval of 7 days, the size of the $\gamma$-ray emitting region should be compact, not larger than $0.05 \mathrm{pc}$, smaller than the dimension of the BLR, $\mathrm{R}_{B L R} \approx 0.12 \mathrm{pc}^{5}$.

In the case of $3 \mathrm{C} 380$ the Lorentz factor estimated by [33] is $\gamma=19.6$. Knowing that $\gamma=\left(1-\beta^{2}\right)^{-1 / 2}$ [2], the Doppler factor of 3C 380 turns out to be $\delta=[\gamma(1-$ $\beta \cos \theta)]^{-1} \approx 5$. On the basis of eq. (1) and considering a time interval of 15 days, the size of the emitting region in 3C 380 is compact, not larger than 0.04 pc, again smaller than the dimension of the $\mathrm{BLR}, \mathrm{R}_{B L R} \approx 0.8 \mathrm{pc}$.

\section{Multiwavelength data}

The next step of our study consisted in building multiwavelength light curves of 3C 207 and 3C 380 (Figures 3 and 4). We utilized both archival and new data (covering the period 2008 February - 2012 November). The new data

\footnotetext{
${ }^{5}$ Following the prescriptions of [32], we calculated the BLR radius assuming that it scales as the square root of the ionizing disk luminosity, $r_{B L R}=10^{17} L_{\text {disk }, 45}^{1 / 2} \mathrm{~cm}$.
} 

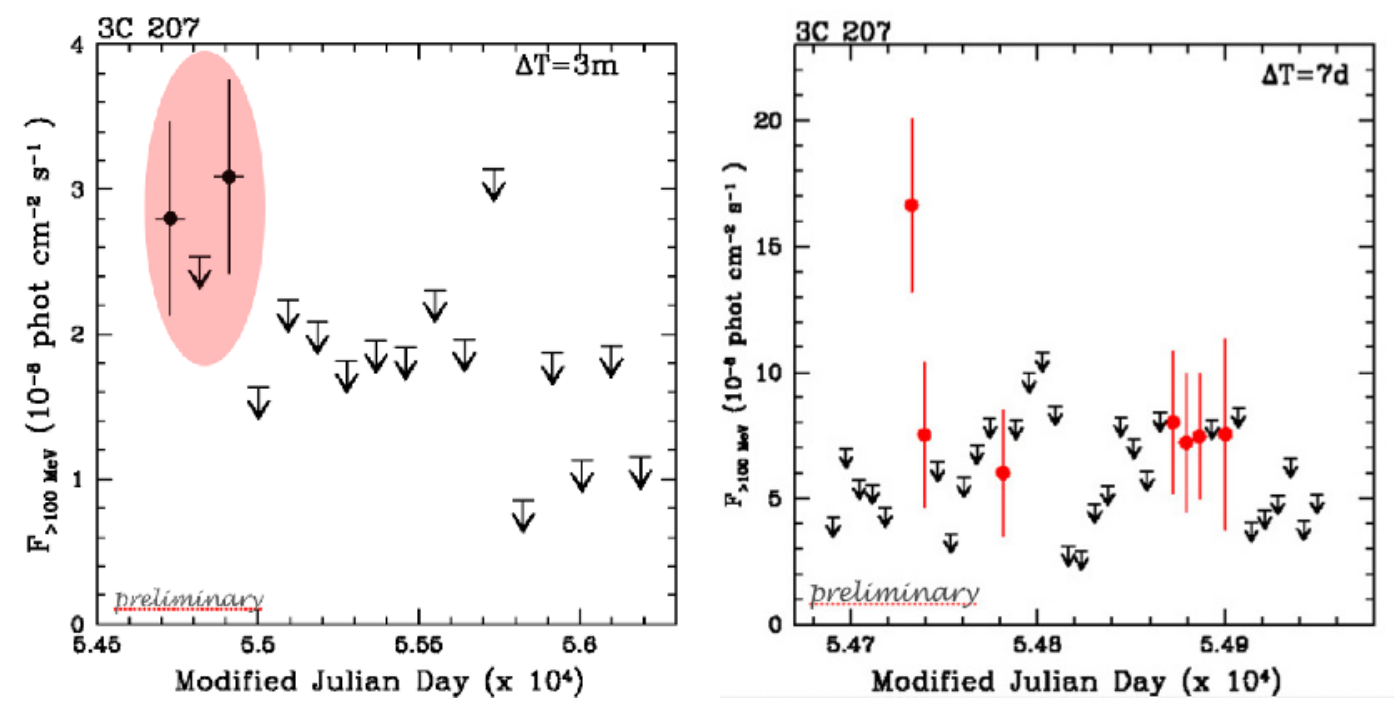

Figure 1. Left panel: LAT light curve of 3C 207 covering 51 months of survey (from 2008 August 4 to 2012 November 4), with a bin time of 3 months. The source is detected only in the first year of observations, while for the rest of the time it is below the LAT sensitivity threshold (black arrows are 2 $\sigma$ upper limits). Right panel: LAT light curve of 3C 207 (from 2008 August 4 to 2009 May 4 ) with a bin time of 1 week. Red points are shown when TS $\geq 10$ while black arrows are $2 \sigma$ upper limits.
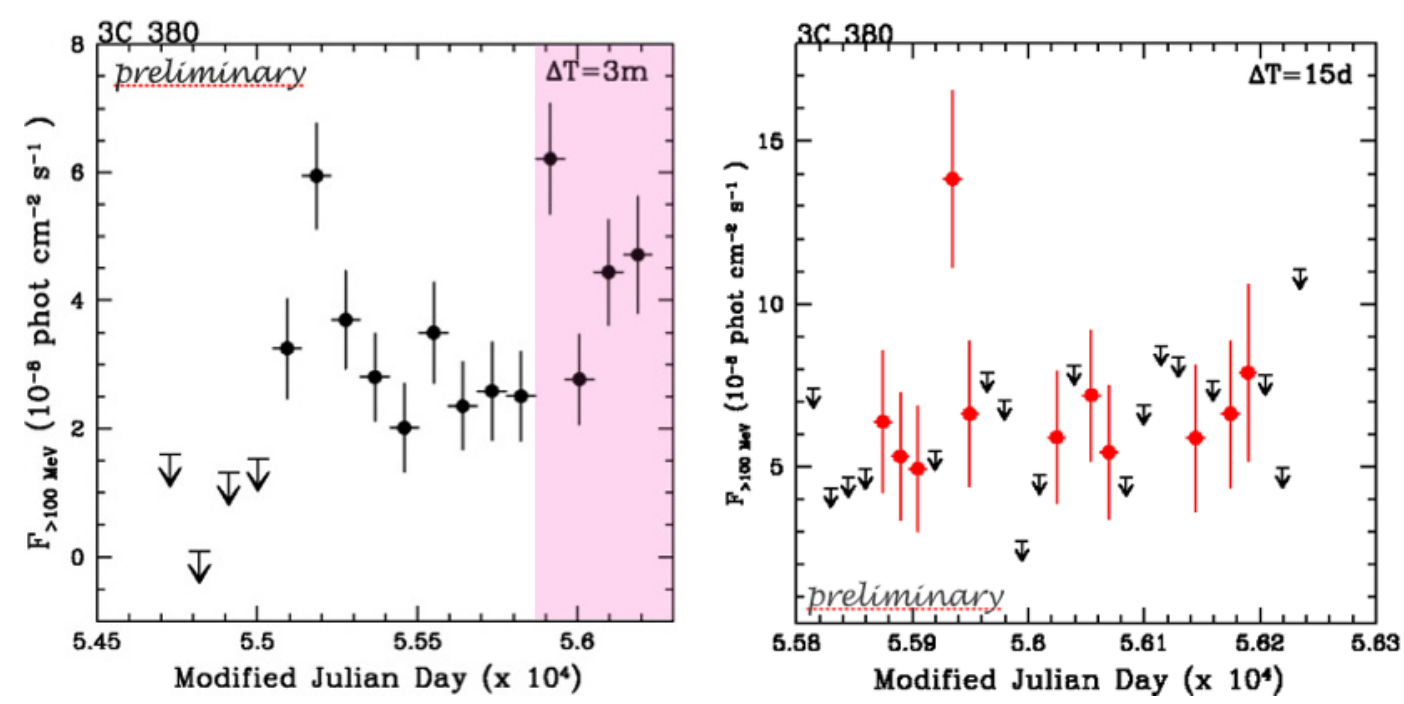

Figure 2. Left panel: LAT light curve of 3C 380 covering 51 months of survey (from 2008 August 4 to 2012 November 4), with a bin time of 3 months. Unlike 3C 207, the source is below the LAT sensitivity threshold during the first year of operations (black arrows are $2 \sigma$ upper limits), and above it for the rest of the time. Two main flares are evident, one occurred between 2009 November 4 and 2010 February 3 and the other one between 2011 November 4 and 2012 February 4. Right panel: LAT light curve of 3C 380 [from 2011 August 10 to 2012 October 31 (pink shadow in the left figure)] with a bin time of 15 days. Red points are shown when TS $\geq 10$ while black arrows are $2 \sigma$ upper limits.

were collected during the TANGO multiwavelength campaign (see Section 1), indicated by the black dotted lines in Figures 3 and 4. The aim was to search for possible correlations between $\gamma$-ray flares and outbursts occurring at other frequencies in an attempt to localize where the high-energy emitting region is located with respect to the central black hole.

In the following a brief overview of the facilities exploited to collect the multiwavelength data of 3C 207 and 3C 380 and the relative analysis is presented:
- X-ray and UV observations have been performed by the Swift X-ray satellite. Both X-ray and UV data have been reduced using the on-line data analysis tools provided by the ASI Science Data Center (ASDC) ${ }^{6}$. The Xray spectra of 3C 207 are always described by a power law with an average photon index $\Gamma=1.8$ absorbed by Galactic column density $\mathrm{N}_{\mathrm{H}, \mathrm{Gal}}=4.2 \times 10^{20} \mathrm{~cm}^{-2}$ [34].

An absorbed power law with $\mathrm{N}_{\mathrm{H}, \mathrm{Gal}}=5.5 \times 10^{20} \mathrm{~cm}^{-2}$ [34] and average $\Gamma=1.65$ is the best fit also for the X-ray

\footnotetext{
${ }^{6} \mathrm{http}: / /$ swift.asdc.asi.it
} 
spectra of 3C 380. In both sources the normalizations (fluxes) change during the entire analyzed period, however the uncertainties are very large and it is difficult to determine the significance of the flux changes.

The UVOT light curve of 3C 207 was built with the filter W1 $(\lambda=2634 \AA)$. A significant increase in the flux starting from 2012 February (during the TANGO campaign, see Figure 3 ) is seen. Two filters were considered to build the UV light curve of 3C 380 (W1 $\lambda=2634 \AA$ and W2 $\lambda=2030 \AA$ ): the state of the source changes in the overall period of observations, however it does not vary significantly during the TANGO campaign.

- Optical data in the R band were collected at the $1.52 \mathrm{~m}$ "G. D. Cassini" telescope of the Astronomical Observatory of Bologna in Loiano, Italy. The photometric data were reduced following standard procedures and using the Image Reduction and Analysis Facility software (IRAF) ${ }^{7}$. The instrumental magnitude of the sources were estimated by applying simple aperture photometry within the APPHOT package in IRAF. Also in this band 3C 207 shows a flux increase (similarly to the UV band) during the TANGO campaign (Figure 3 ).

- Radio data: public data at $15 \mathrm{GHz}$ and $230 \mathrm{GHz}$ were used to cover the radio and mm bands. Indeed, 3C 207 and 3C 380 are part of the MOJAVE (Monitoring Of Jets in Active galactic nuclei with VLBA Experiments) program ${ }^{8}$ with the Very Long Baseline Array (VLBA) and are also included in the calibrator list of the Submillimeter Array (SMA) ${ }^{9}$ (for details see [35]). In both sources slow flux variations on the time scale of years are observed.

Multifrequency monitoring is a difficult task requiring a huge effort to collect simultaneous data necessary to find possible connections among different wavebands. Our campaign, although just at the beginning, has provided some interesting information. For example, a temporal coincidence between the $\gamma$-ray and mm flares is clearly evident in 3C 380 (Figure 4 lower panel) indicating a possible co-spatiality of these events. It is also worth noting that the flux decay is very similar in both bands. If this is an indication of a jet perturbation the analysis of high-resolution radio images (at $15 \mathrm{GHz}$ or $43 \mathrm{GHz}$ for example) should reveal modifications of the jet allowing a spatial localization of the event. The analysis of the $15 \mathrm{GHz}$ MOJAVE images available from the public archive is ongoing and the results will be presented in Torresi et al. (2013 in prep.).

In the case of 3C 207 we observed a simultaneous increase of the optical and UV fluxes during the TANGO campaign (Figure 3 ) not accompanied by any $\gamma$-ray activity (the GeV flux is below the LAT threshold). This could indicate that a perturbation is occurring only in the disk. However radio and $\mathrm{mm}$ data, surely related to the jet, are

\footnotetext{
${ }^{7}$ http://iraf.noao.edu/

${ }^{8}$ https://www.physics.purdue.edu/astro/mojave/

${ }^{9}$ http://sma1.sma.hawaii.edu/callist/callist.html
}

too sparse to rule out any possible link between thermal and non-thermal components.

\section{Summary}

In this work we presented the preliminary results of the $\gamma$-ray and multiwavelength study of 3C 207 and 3C 380, the only two SSRQ of our MAGN sample. The study of the $\gamma$-ray light curves pointed out that the high-energy photons are produced in very compact regions with a size of a few $10^{-2} \mathrm{pc}$, well below the BLR dimension.

The preliminary results of the (still ongoing) TANGO multiwavelength campaign are very promising: in the case of 3C 380 we could catch a simultaneous outburst and successive flux decay of the $\mathrm{mm}$ and $\gamma$-ray light curves pointing to a possible co-spatiality of the events. This triggered a deep study of the MOJAVE radio maps, which is underway, looking for spatial jet modifications. In the case of 3C 207 we did not observe any $\gamma$-ray outburst during the simultaneous increase of the optical and UV fluxes in 2012. This optical/UV flux change could suggest a perturbation in the disk rather than in the jet. In order to be more conclusive on this issue, we have to wait for more data at low frequencies to trace the behavior of non-thermal photons.

\section{Acknowledgments}

The Fermi-LAT Collaboration acknowledges the generous support of a number of agencies and institutes that have supported the Fermi-LAT Collaboration. These include the National Aeronautics and Space Administration and the Department of Energy in the United States, the Commissariat à l'Energie Atomique and the Centre National de la Recherche Scientifique / Institut National de Physique Nucléaire et de Physique des Particules in France, the Agenzia Spaziale Italiana and the Istituto Nazionale di Fisica Nucleare in Italy, the Ministry of Education, Culture, Sports, Science and Technology (MEXT), High Energy Accelerator Research Organization (KEK) and Japan Aerospace Exploration Agency (JAXA) in Japan, and the K. A. Wallenberg Foundation, the Swedish Research Council and the Swedish National Space Board in Sweden. Additional support for science analysis during the operations phase is gratefully acknowledged from the Istituto Nazionale di Astrofisica in Italy and the Centre National d'Études Spatiales in France.

E. T. and P. G. would like to greatly thank Ivan Bruni, Silvia Galleti, Roberto Gualandi and Valentina Zitelli for the kind technical support at the "G. D. Cassini" telescope in Loiano.

\section{References}

[1] Fanaroff, B. L. \& Riley, J. M., MNRAS, 167, 31 (1974)

[2] Urry, C. M., \& Padovani, P., PASP, 107, 803 (1995)

[3] Grandi, P., in Proc. Workshop on High Energy Phenomena in Relativistic Outflows III (HEPRO III), ed. J. M. Paredes et al. (2011) 


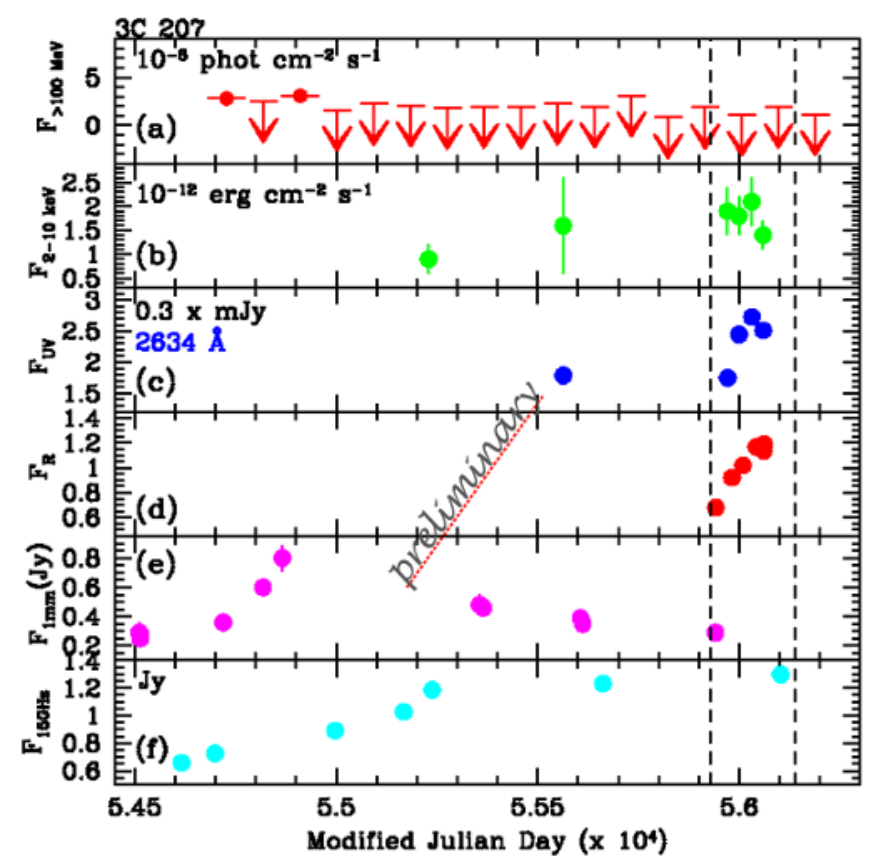

Figure 3. Multiwavelength light curve of 3C 380. (a): 51-month LAT light curve (bin time=3 months); (b)-(c): X-ray and UV $(\lambda=2634 \AA$ ) data from the Swift X-ray satellite; $(d)$ : optical R-band data collected at the "G. D. Cassini" telescope in Loiano; $(e)$ : $230 \mathrm{GHz}$ data from the SMA public archive; $(e): 15 \mathrm{GHz}$ data from the MOJAVE program.

[4] Atwood, W. B., et al., ApJ, 697, 1071 (2009)

[5] Abdo, A. A., et al., ApJ, 720, 912 (2010a)

[6] Tavecchio, F., Ghisellini, G., Bonnoli, G., \& Ghirlanda, G., MNRAS, 405, L95 (2010)

[7] Poutanen, J., Stern, B., ApJ, 717, 118 (2010)

[8] Marscher, A. P., et al., Nature, 452, 966 (2008)

[9] Marscher A. P., et al., ApJ, 710, L126 (2010)

[10] Jorstad, S. G., et al., ApJS, 134, 181 (2001)

[11] Jorstad, S. G., et al., ApJ, 715, 362 (2010)

[12] Lähteenmäki, A. \& Valtaoja, E., ApJ, 590, 95 (2003)

[13] Agudo, I., et al., ApJ, 726, L13 (2011a)

[14] Agudo, I., et al., ApJ, 735, L10 (2011b)

[15] Abdo, A. A., Ackermann, M., Ajello, M., et al., Science, 328, 725 (2010b)

[16] Grandi, P., Torresi, E., \& Stanghellini, C., ApJL, 715, L3 (2012)

[17] Chatterjee, R., Marscher, A. P., Jorstad, S. G., et al., ApJ, 734, 43 (2011)

[18] Laing, R. A., Riley, J. M. \& Longair, M. S., MNRAS, 204, 151 (1983)

[19] Wall, J. V. \& Peacock, J. A., MNRAS, 216, 173 (1985)

[20] Adelman-McCarthy, J. K., et al. 2009, ApJS, 182, 543
[21] Bogers, W. J., Hes, R., Barthel, P. D., Zensus, J. A., A\&AS, 105, 91 (1994)

[22] Brunetti, G., Bondi, M., Comastri, A., Setti, G., A\&A, 381, 795 (2002)

[23] Fanti, R., Fanti, C., et al., A\&A, 231, 333 (1990)

[24] Murphy, D. W., Browne, I. W. A., Perley, R. A., MNRAS, 264, 298 (1993)

[25] de Vries, W. A., et al., 1997, ApJS, 110, 191 (1997)

[26] Pushkarev, A. B., et al., A\&A, 507, 33 (2009)

[27] Polatidis, A. G., Wilkinson, P. N., MNRAS, 294, 327 (1998)

[28] O’Dea, C. P., et al., AJ, 117, 1143 (1999)

[29] Nolan, P. L., et al., ApJS, 199, 31 (2012)

[30] Mattox, J. R., Bertsch, D. L., Chiang, J., et al., ApJ, 461, 396 (1996)

[31] Lister, M. L., et al., AJ in press, arXiv:1308.2713 (2013)

[32] Ghisellini, G., Tavecchio, F., MNRAS, 387, 1669 (2008)

[33] Pushkarev, A. B., et al., A\&A, 507, 33 (2009)

[34] Kalberla, P. M. W., et al., A\&A, 440, 775 (2005)

[35] Gurwell,M. A., et al., in ASP Conf. Ser. 375, From Z-machines to ALMA: (Sub)Millimeter Spectroscopy of Galaxies, ed. A. J. Baker et al. San Francisco, CA, 234 (2007) 

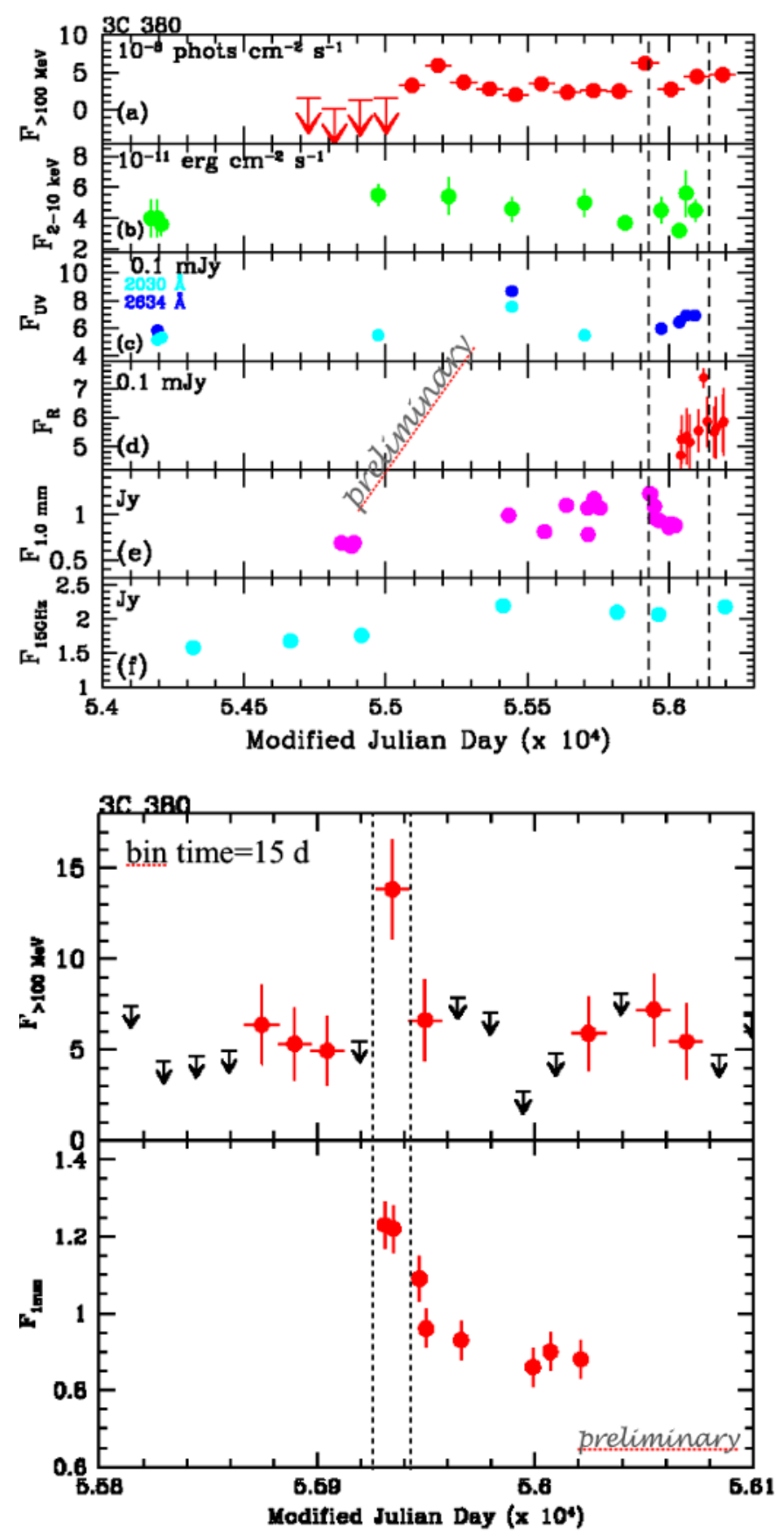

Figure 4. Upper panel: Multiwavelength light curve of 3C 380. (a): 51-month LAT light curve (bin time=3 months); (b)-(c): X-ray and UV data from the Swift X-ray satellite; $(d)$ : optical R-band data collected at the "G. D. Cassini" telescope in Loiano; $(e): 230 \mathrm{GHz}$ data from the SMA public archive; $(e): 15 \mathrm{GHz}$ data from the MOJAVE program. Lower panel: Top- Gamma-ray light curve of 3C 380 from 2011 November 2 to 2012 March 1 (bin time=15 days). The highest GeV activity was reached on January 2012 (from 1st to 16th). Bottom- 1 mm SMA observations in the temporal interval 2012 January 5 - 2012 April 4. 